\title{
Spatial distribution of soft-bottom polychaete annelids in the Ensenada de Baiona (Ría de Vigo, Galicia, north-west Spain)
}

\author{
JUAN MOREIRA, PATRICIA QUINTAS and JESÚS S. TRONCOSO \\ Departamento de Ecoloxía e Bioloxía Animal, Facultade de Ciencias, Campus de Lagoas-Marcosende s/n, \\ Universidade de Vigo, 36200 Vigo, Spain. E-mail: moreira@uvigo.es
}

\begin{abstract}
SUMMARY: The spatial distribution of the polychaete fauna in the subtidal soft-bottoms of the Ensenada de Baiona (Galicia, NW Spain) was studied by means of quantitative sampling and multivariate analyses. Several faunistic assemblages were determined, which were closely related to the sediment distribution pattern in the inlet. The coarse-medium sand fauna was mainly dominated by syllids and pisionids. Finer sediments (fine sand to mud) showed a greater abundance of depositfeeding species, mostly spionids, cirratulids and capitellids. The greatest number of species and diversity were found in mixed sediments with medium and fine sand. Organically enriched muds around Baiona harbour have an impoverished fauna, with a high dominance of Cossura pygodactylata Jones, 1956.
\end{abstract}

Keywords: Polychaeta, soft-bottoms, distribution, multivariate analysis, Ensenada de Baiona, Spain, Atlantic Ocean.

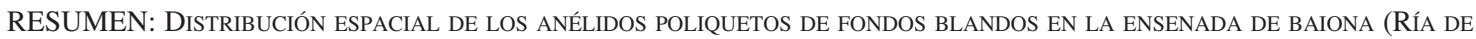
Vigo, Galicia, NoROESTE DE EsPAÑa). - La distribución espacial de la fauna de poliquetos de fondos blandos submareales de la ensenada de Baiona (Galicia, NW España) ha sido estudiada mediante técnicas de muestreo cuantitativo. Los análisis multivariantes diferenciaron varias asociaciones faunísticas, que coinciden en gran medida con los patrones de distribución sedimentaria en el área estudiada. La fauna de arenas gruesas y medias está dominada principalmente por sílidos y pisiónidos, mientras que los sedimentos más finos (arenas finas a fangos) muestran una mayor abundancia de organismos depositívoros como espiónidos, cirratúlidos y capitélidos. Se han observado los mayores valores de riqueza específica y diversidad en sedimentos mixtos de arena fina y media. Los fangos orgánicamente enriquecidos del puerto de Baiona muestran una fauna empobrecida con una gran dominancia de Cossura pygodactylata.
\end{abstract}

Palabras clave: Polychaeta, fondos blandos, distribución, análisis multivariante, ensenada de Baiona, España, Océano Atlántico.

\section{INTRODUCTION}

The Galician "rías" are considered to be complex and special estuarine systems, with great economic and social importance (fisheries, bivalve culture on rafts, shellfish resources) (López-Jamar et al., 1984). A scientific study of the different aspects of the environment is thus necessary for appropriate management of resources. In this context, the study of the composition and distribution of the benthic assemblages is of great interest, because they have been considered as good indicators of marine bottom conditions (Bellan, 1967; Gray and Mirza, 1979). The marine benthos of the rías has been extensively studied over recent years. In the case of the Ría de Vigo, however, studies of soft-bottom benthic communities are scarce when compared to other rías (Viéitez, 1976; López-Jamar and Cal, 1990; Abella et al., 1996). Furthermore, the softbottom polychaete fauna of this ría is poorly known, 
both in terms of taxonomic and sinecological studies (Viéitez, 1976; Planas, 1986).

The Ensenada de Baiona is a small inlet located on the southern margin of the Ría de Vigo, whose sedimentary composition has been partially altered by the construction of a harbour jetty during the 1970's (Alejo and Vilas, 1987). Several taxonomic works have been recently published on its polychaete fauna (Moreira et al., 2000a, b; Parapar et al., 2000), but there is an evident lack of ecological studies. Thus, the main objective of this paper is to characterize the composition and distribution of the polychaete fauna on subtidal soft-bottoms of the Ensenada de Baiona. We also study the possible relation between several environmental variables and faunal distribution patterns. The present study was carried out after the construction of the already mentioned jetty and prior to the location of mussel culture rafts in the middle of the inlet, and therefore can provide a baseline for further comparative studies.

\section{MATERIAL AND METHODS}

\section{Sampling and laboratory procedures}

The soft-bottom polychaete fauna was quantitatively sampled at 21 sites during December 1995 (Fig. 1). Five replicates were taken at each site, using a Van Veen grab $\left(0.056 \mathrm{~m}^{2}\right)$. Samples were sieved through a $0.5 \mathrm{~mm}$ mesh and fixed in $10 \%$ buffered formalin for later sorting and identification of the fauna. An additional sediment sample was also taken at each site to measure in situ $\mathrm{pH}$ and temperature, particle size composition, carbonate and organic matter content. The following granulometric fractions were considered: gravel (GR, >2 $\mathrm{mm}$ ), very coarse sand (VCS, $2-1 \mathrm{~mm}$ ), coarse sand (CS, 1-0.5 mm), medium sand (MS, 0.5-0.25 mm), fine sand (FS, 0.25-0.125 mm), very fine sand (VFS, $0.125-0.063 \mathrm{~mm})$, and silt/clay $(<0.063 \mathrm{~mm})$. Median grain size $\left(\mathrm{Q}_{50}\right)$, sort coefficient $\left(\mathrm{S}_{\mathrm{o}}\right)$ (Trask, 1932), skewness (SK) and kurtosis (KG) were also determined for each sample. Sedimentary types were characterized according to Junoy and Viéitez (1989). Carbonate content (\%) was estimated by treatment of the sample with hydrochloric acid. Total organic matter content (TOM, \%) was estimated from the weight loss on combustion at $450^{\circ} \mathrm{C}$ for 4 hours.
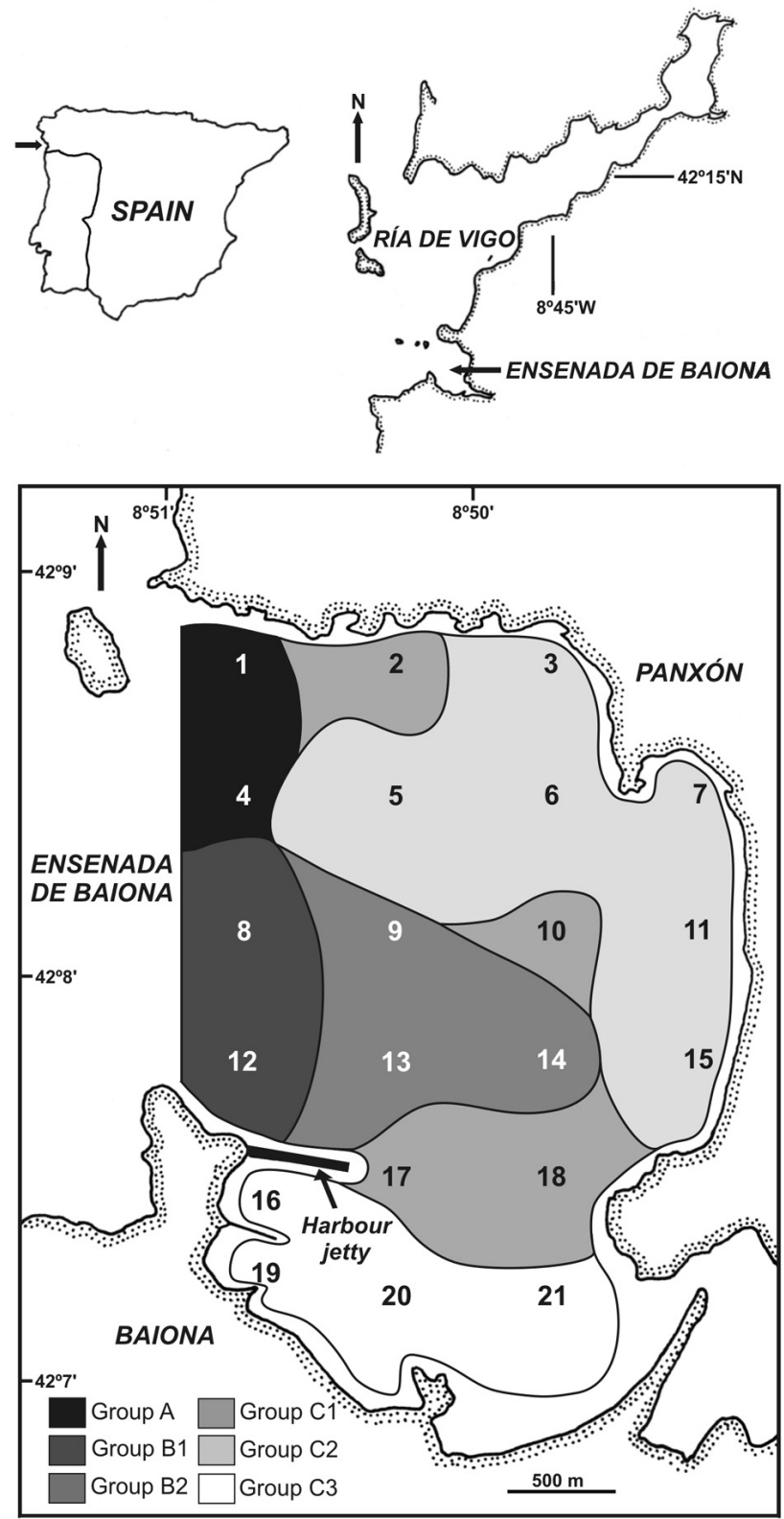

FIG. 1. - Map showing the location of the Ensenada de Baiona and distribution of polychaete assemblages.

\section{Data analyses}

The following univariate measures were calculated for each site: total abundance of all polychaetes $(\mathrm{N})$, total number of species $(\mathrm{S})$, the Shannon-Wiener diversity index $\left(\mathrm{H}^{\prime}\right.$, as $\left.\log _{2}\right)$, and Pielou's evenness $(\mathrm{J})$. For any given site, species with $\geq 4 \%$ of total abundance were considered as dominant (Field et al., 1982). Polychaete assemblages were determined through non-parametric multivariate techniques as described by Field et al. (1982), using the PRIMER v5.0 software package (Clarke and Warwick, 1994). Similarities between 
sites were determined using the Bray-Curtis similarity coefficient. Species abundance data were transformed by the fourth root to downweight the contribution to similarity of the most abundant species. A classification of the sites was performed from the similarity matrix by cluster analysis based on the group-average sorting algorithm and an ordination by means of non-metric multidimensional scaling (MDS). Differences in faunistic composition between samples were tested using the 1-way ANOSIM test. The species present in each assemblage were classified according to the values of the Constancy and Fidelity indexes (Cabioch, 1968).

The possible relationship between polychaete distribution and measured environmental variables was determined using the BIO-ENV procedure (PRIMER), and the canonical correspondence analysis (CCA) using CANOCO v4.02 (ter Braak, 1988). Forward selection was employed in the latter to detect which variables explained the most variance in the species data. All environmental variable values were previously transformed by $\log (\mathrm{x}+1)$ and then normalised due to the different units of measurement. The following variables were considered in these analyses: TOM, granulometric fractions (GR, CS, MS, FS, VFS), median grain size, sorting coefficient, skewness, kurtosis, temperature and depth, while carbonates, VCS, silt/clay and $\mathrm{pH}$ were excluded due to their high correlation with other variables $(r>0.9, p<0.01)$. Sites 1 and 4 were discarded because of their stony nature, which could make interpretation of the analyses difficult and too small a sample from site 4 .

\section{RESULTS}

Sediments were predominantly sandy (Table 1), ranging from coarse sand to fine muddy sand, with small amounts of silt/clay and organic matter. Muds and sandy muds were located in the Baiona harbour area, with TOM content ranging from moderate to high $(2.8-12.0 \%)$, while gravels were restricted to the northern outer part of the inlet (Fig. 1).

Polychaetes were the dominant infaunal taxon, accounting for $34.5 \%$ of total abundance. A total of 15,453 polychaete specimens were collected, belonging to 133 species in 39 families. Syllids and spionids were the dominant families in terms of species number, with 26 and 14 respectively. The spionids Spiophanes bombyx (Claparède, 1870) and Prionospio fallax Söderström, 1920, the syllids Streptosyllis websteri Southern, 1914, Parapionosyllis cabezali Parapar, San Martín and Moreira, 2000, Exogone hebes (Webster and Benedict, 1884) and E. naidina Örsted, 1845, the pisionid Pisione parapari Moreira, Quintas and Troncoso, 2000, the polygordiid Polygordius appendiculatus Fraipont, 1887 and the cirratulid Chaetozone setosa Malmgren, 1867 accounted for $50 \%$ of all polychaetes.

TABLE 1. - Coordinates and physical characteristics of the sampling sites. $\mathrm{Q}_{50}$, median grain size; $\mathrm{CO}_{3}{ }^{=}$, carbonate content; TOM, total organic matter content.

\begin{tabular}{|c|c|c|c|c|c|c|c|c|c|c|c|}
\hline Site & Position (N) & Position (W) & $\begin{array}{l}\text { Depth } \\
\text { (m) }\end{array}$ & $\begin{array}{c}\text { Gravel } \\
(\%)\end{array}$ & $\begin{array}{l}\text { Sand } \\
(\%)\end{array}$ & $\begin{array}{l}\text { Silt/Clay } \\
(\%)\end{array}$ & $\begin{array}{c}\mathrm{Q}_{50} \\
(\mathrm{~mm})\end{array}$ & Sedimentary type & Sorting & $\begin{array}{c}\mathrm{CO}_{3}= \\
(\%)\end{array}$ & $\begin{array}{c}\text { TOM } \\
(\%)\end{array}$ \\
\hline 1 & $42^{\circ} 08^{\prime} 50^{\prime \prime} \mathrm{N}$ & $08^{\circ} 50^{\prime} 52^{\prime \prime} \mathrm{W}$ & 7 & 94.23 & 5.74 & 0.03 & 5.00 & Gravel & Moderate & 24.26 & 1.48 \\
\hline 2 & $42^{\circ} 08^{\prime} 50^{\prime \prime} \mathrm{N}$ & $08^{\circ} 50,15^{\prime,} \mathrm{W}$ & 7 & 3.52 & 89.96 & 6.52 & 0.13 & Muddy sand & Moderate & 32.39 & 1.91 \\
\hline 3 & $42^{\circ} 08^{\prime} 50^{\prime \prime} \mathrm{N}$ & $08^{\circ} 49^{\prime} 44^{\prime \prime} \mathrm{W}$ & 4 & 0.01 & 87.19 & 12.80 & 0.09 & Muddy sand & Mod. well sorted & 29.67 & 2.27 \\
\hline 4 & $42^{\circ} 08^{\prime} 30^{\prime \prime} \mathrm{N}$ & $08^{\circ} 50^{\prime} 52^{\prime}, \mathrm{W}$ & 12 & - & - & - & - & - & - & - & - \\
\hline 5 & $42^{\circ} 08^{\prime} 30^{\prime}, \mathrm{N}$ & $08^{\circ} 50^{\prime} 15^{\prime,} \mathrm{W}$ & 11 & 0.15 & 88.40 & 11.45 & 0.09 & Muddy sand & Mod. well sorted & 34.37 & 1.70 \\
\hline 6 & $42^{\circ} 08^{\prime} 30^{\prime \prime} \mathrm{N}$ & $08^{\circ} 49^{\prime} 44^{\prime,} \mathrm{W}$ & 7 & 0.09 & 90.66 & 9.25 & 0.09 & Muddy sand & Mod. well sorted & 37.59 & 2.10 \\
\hline 7 & $42^{\circ} 08^{\prime} 30^{\prime \prime} \mathrm{N}$ & $08^{\circ} 49^{\prime} 13^{\prime \prime} \mathrm{W}$ & 3 & 0.04 & 96.44 & 3.53 & 0.15 & Fine sand & Moderate & 48.33 & 2.05 \\
\hline 8 & $42^{\circ} 08^{\prime} 10^{\prime \prime} \mathrm{N}$ & $08^{\circ} 50^{\prime} 52^{\prime \prime} \mathrm{W}$ & 12 & 10.29 & 88.40 & 1.31 & 0.83 & Coarse sand & Moderate & 68.94 & 1.48 \\
\hline 9 & $42^{\circ} 08^{\prime} 10^{\prime \prime} \mathrm{N}$ & $08^{\circ} 50^{\prime} 15^{, \prime} \mathrm{W}$ & 10 & 1.24 & 95.70 & 3.06 & 0.35 & Medium sand & Moderate & 82.67 & 2.28 \\
\hline 10 & $42^{\circ} 08^{\prime} 10^{\prime \prime} \mathrm{N}$ & $08^{\circ} 49^{\prime} 44^{\prime \prime} \mathrm{W}$ & 8 & 5.84 & 88.94 & 5.22 & 0.14 & Muddy sand & Moderate & 49.13 & 2.20 \\
\hline 11 & $42^{\circ} 08^{\prime} 10^{\prime \prime} \mathrm{N}$ & $08^{\circ} 49^{\prime} 13^{\prime \prime} \mathrm{W}$ & 3 & 0.18 & 84.28 & 15.54 & 0.10 & Muddy sand & Mod. well sorted & 44.20 & 2.58 \\
\hline 12 & $42^{\circ} 07^{\prime} 50^{\prime \prime} \mathrm{N}$ & $08^{\circ} 50^{\prime} 52^{\prime \prime} \mathrm{W}$ & 9 & 8.27 & 91.71 & 0.03 & 0.90 & Coarse sand & Moderate & 61.31 & 1.32 \\
\hline 13 & $42^{\circ} 07^{\prime} 50^{\prime \prime} \mathrm{N}$ & $08^{\circ} 50^{\prime} 15^{,}, \mathrm{W}$ & 8 & 0.44 & 96.47 & 3.09 & 0.34 & Medium sand & Moderate & 79.68 & 2.07 \\
\hline 14 & $42^{\circ} 07^{\prime} 50^{\prime \prime} \mathrm{N}$ & $08^{\circ} 49^{\prime} 44^{\prime \prime} \mathrm{W}$ & 9 & 1.14 & 95.70 & 3.16 & 0.31 & Medium sand & Moderate & 80.35 & 2.18 \\
\hline 15 & $42^{\circ} 07^{\prime} 50^{\prime \prime} \mathrm{N}$ & $08^{\circ} 49^{\prime} 13^{\prime,} \mathrm{W}$ & 4 & 0.03 & 95.83 & 4.14 & 0.14 & Fine sand & Moderate & 45.00 & 2.32 \\
\hline 16 & $42^{\circ} 07^{\prime} 30^{\prime \prime} \mathrm{N}$ & $08^{\circ} 50^{\prime} 45^{\prime,} \mathrm{W}$ & 4 & 0.04 & 9.85 & 90.11 & 0.02 & Mud & Poor & 5.81 & 12.05 \\
\hline 17 & $42^{\circ} 07^{\prime} 30^{\prime \prime} \mathrm{N}$ & $08^{\circ} 50^{\prime} 15^{\prime,} \mathrm{W}$ & 7 & 9.63 & 84.18 & 6.19 & 0.23 & Muddy sand & Moderate & 72.91 & 3.18 \\
\hline 18 & $42^{\circ} 07^{\prime} 30^{\prime \prime} \mathrm{N}$ & $08^{\circ} 49^{\prime} 44^{\prime,} \mathrm{W}$ & 8 & 2.11 & 94.08 & 3.82 & 0.23 & Fine sand & Moderate & 75.33 & 2.48 \\
\hline 19 & $42^{\circ} 07^{\prime} 19, \times \mathrm{N}$ & $08^{\circ} 50^{\prime} 45^{\prime,} \mathrm{W}$ & 2 & 2.53 & 22.95 & 74.52 & 0.02 & Mud & Poor & 7.11 & 8.45 \\
\hline 20 & $42^{\circ} 07^{\prime} 10^{\prime \prime} \mathrm{N}$ & $08^{\circ} 50^{\prime} 15^{\prime,} \mathrm{W}$ & 3 & 0.17 & 21.66 & 78.17 & 0.02 & Mud & Poor & 6.85 & 7.28 \\
\hline 21 & $42^{\circ} 07^{\prime} 10^{\prime \prime} \mathrm{N}$ & $08^{\circ} 49^{\prime} 44^{\prime \prime} \mathrm{W}$ & 3 & 0.19 & 50.61 & 49.20 & 0.06 & Sandy mud & Poor & 4.36 & 2.82 \\
\hline
\end{tabular}


TABLE 2. - Total number of species per $0.28 \mathrm{~m}^{2}(\mathrm{~S})$, total abundance per $0.28 \mathrm{~m}^{2}(\mathrm{~N})$, Shannon-Wiener's diversity index $\left(\mathrm{H}^{\prime}, \log _{2}\right)$ and Pielou's evenness (J) for each sampling site.

\begin{tabular}{lcccc}
\hline Site & S & N & H' & J \\
\hline $\mathbf{1}$ & 19 & 919 & 2.49 & 0.59 \\
$\mathbf{2}$ & 49 & 1328 & 4.21 & 0.75 \\
$\mathbf{3}$ & 14 & 134 & 2.89 & 0.76 \\
$\mathbf{4}$ & 43 & 403 & 4.32 & 0.80 \\
$\mathbf{5}$ & 22 & 443 & 2.72 & 0.61 \\
$\mathbf{6}$ & 25 & 366 & 3.42 & 0.74 \\
$\mathbf{7}$ & 16 & 70 & 3.23 & 0.81 \\
$\mathbf{8}$ & 36 & 1990 & 3.68 & 0.71 \\
$\mathbf{9}$ & 28 & 690 & 3.34 & 0.69 \\
$\mathbf{1 0}$ & 39 & 572 & 4.21 & 0.80 \\
$\mathbf{1 1}$ & 17 & 134 & 2.84 & 0.69 \\
$\mathbf{1 2}$ & 33 & 1820 & 3.48 & 0.69 \\
$\mathbf{1 3}$ & 25 & 808 & 2.04 & 0.44 \\
$\mathbf{1 4}$ & 31 & 1120 & 2.25 & 0.45 \\
$\mathbf{1 5}$ & 24 & 684 & 2.92 & 0.64 \\
$\mathbf{1 6}$ & 13 & 228 & 1.52 & 0.41 \\
$\mathbf{1 7}$ & 39 & 668 & 4.00 & 0.76 \\
$\mathbf{1 8}$ & 44 & 558 & 4.02 & 0.74 \\
$\mathbf{1 9}$ & 30 & 592 & 3.99 & 0.81 \\
$\mathbf{2 0}$ & 29 & 357 & 3.76 & 0.77 \\
$\mathbf{2 1}$ & 31 & 1569 & 3.34 & 0.67 \\
\hline
\end{tabular}

The number of polychaete species per site ranged from 13 to 49 (Table 2). Maximum densities of polychaetes were found at sites 8 and 12, and the lowest at sites 7,3 and 11 . The highest diversity was recorded at sites 4, 2 and $10\left(\mathrm{H}^{\prime}>4.20\right)$ in contrast to that found at St. 16 (H': 1.52). The lowest value of evenness was found at site 16 (J: 0.41), in which the cossurid Cossura pygodactylata was very dominant.

\section{Faunal assemblages}

The ANOSIM test revealed significant differences in faunistic composition between all sites, except 11 and 16 (r: $0.364, \mathrm{p}>0.05$ ). The cluster analysis showed the presence of three major groups of sites at a similarity level of $30 \%$ : group A (gravel bottoms), group B (coarser sandy sediments) and group $\mathrm{C}$ (fine sand to muddy sediments). At a similarity level of $50 \%$, group B could be divided in two further groups, B1 (coarse sand bottoms) and B2 (medium sand), while group $\mathrm{C}$ was divided into three groups: $\mathrm{C} 1$ (mixed fine sand bottoms at the centre of the inlet), $\mathrm{C} 2$ (fine sand bottoms at the northern and eastern margins of the inlet) and $\mathrm{C} 3$ (muddy bottoms around the Baiona harbour). This classification mostly agrees with the results obtained through MDS analysis. The abiotic and faunistic characteristics of the six assemblages are summarized and their locations in the Ensenada de Baiona are shown (Table 3, Fig. 1).
Group A comprised two sites $(1,4)$ with a low similarity. The stony-gravel nature of these bottoms favoured the presence of species such as the serpulids Pomatoceros spp. and Vermiliopsis striaticeps (Grube, 1862), while Polygordius lacteus Schneider, 1868 and Amphiglena mediterranea (Leydig, 1851) were found only here.

Group B1 was comprised of coarse sand bottoms (sites 8,12 ), characterized by highest densities of the inlet, and were mainly due to the abundance of the pisionids Pisione parapari and $P$. remota (Southern, 1914), several syllids (Parapionosyllis cabezali, Pionosyllis lamelligera Saint-Joseph, 1856, Sphaerosyllis taylori Perkins, 1981, Exogone naidina) and Polygordius appendiculatus. Group B2 (sites $9,13,14$ ) shared a similar faunistic composition with $\mathrm{B} 1$, but several species such as Pisione remota, Polygordius appendiculatus, Parapionosyllis cabezali, Pionosyllis lamelligera and Sphaerosyllis taylori showed lesser abundances in this assemblage. The syllid Streptosyllis websteri reached its highest densities in this group, which was also characterized by the presence of Paradoneis lyra (Southern, 1914) (constant, preferential), Clymenura sp. (constant, exclusive) and Caulleriella bioculata (Keferstein, 1862) (constant, occasional).

Group C1 comprised 4 sites $(2,10,17,18)$. Sediments were characterized as finemuddy sands. This group exhibited the highest total number of species and diversity amongst all assemblages. Several numerically dominant species were also present in medium sand bottoms (Streptosyllis websteri, Caulleriella bioculata) and other finer sediments (Capitella capitata (Fabricius, 1780), Spiophanes bombyx, Prionospio fallax, Spio decoratus Bobretzky, 1871, Glycera tridactyla Schmarda, 1861), while Chaetozone setosa (constant, preferential), Galathowenia oculata (Zaks, 1922) (constant, preferential) and Caulleriella alata (Southern, 1914) (constant, preferential) showed a greater presence in this assemblage. Group C2 was composed by six sites $(3,5,6,7,11,15)$, that were in general shallower in depth than those of group C1 and with a greater percentage of very fine sand and silt/clay. This assemblage was poorer than $\mathrm{C} 1$ both in terms of number of species and abundance. The spionid Spiophanes bombyx accounted for more than $40 \%$ of the total number of individuals of the assemblage. These bottoms were also characterized by the presence of Magelona johnstoni Fiege, 
TABLE 3. - Summary of biotic and physical characteristics of the six assemblages derived from multivariate analysis (values: mean ? standard deviation). Species that add up to $\geq 70 \%$ of the total abundance in each assemblage are listed in order of dominance. S, number of species per $0.28 \mathrm{~m}^{2}$; N, number of individuals per $0.28 \mathrm{~m}^{2}$; H', Shannon-Wiener diversity; J, Pielou's evenness; TOM, total organic matter; CO ${ }_{3}$, carbonate content; $\mathrm{Q}_{50}$, median grain size. Constancy: CO, constant; VC, very common; CM, Common. Fidelity: EX, exclusive; EL, elective; $\mathrm{PR}$, preferential; AC, accessory; OC, occasional.

\begin{tabular}{|c|c|c|c|c|c|c|}
\hline & $\begin{array}{l}\text { Group A } \\
(1,4)\end{array}$ & $\begin{array}{l}\text { Group B1 } \\
(8,12)\end{array}$ & $\begin{array}{l}\text { Group B2 } \\
(9,13,14)\end{array}$ & $\begin{array}{l}\text { Group C1 } \\
(2,10,17,18)\end{array}$ & $\begin{array}{l}\text { Group C2 } \\
(3,5,6,7,11,15)\end{array}$ & $\begin{array}{l}\text { Group C3 } \\
(16,19,20,21)\end{array}$ \\
\hline $\begin{array}{l}\text { Dominant } \\
\text { taxa }\end{array}$ & $\begin{array}{l}\text { Amphiglena medite- } \\
\text { rranea }(\mathrm{CM} / \mathrm{EX}) \\
\text { Polygordius lacteus } \\
\text { (CM/EX) } \\
\text { Pomatoceros } \\
\text { lamarcki }(\mathrm{CO} / \mathrm{EX}) \\
\text { Pomatoceros } \\
\text { triqueter }(\mathrm{CO} / \mathrm{EX}) \\
\text { Heteromastus } \\
\text { filiformis }(\mathrm{CM} / \mathrm{OC})\end{array}$ & $\begin{array}{l}\text { Pisione parapari } \\
\text { (CO/AC) } \\
\text { Polygordius appendi- } \\
\text { culatus }(\mathrm{CO} / \mathrm{AC}) \\
\text { Parapionosyllis } \\
\text { cabezali }(\mathrm{CO} / \mathrm{AC}) \\
\text { Pisione remota } \\
\text { (CO/AC) } \\
\text { Pionosyllis } \\
\text { lamelligera } \\
\text { (CO/AC) }\end{array}$ & $\begin{array}{l}\text { Streptosyllis websteri } \\
\text { (CO/OC) } \\
\text { Pisione parapari } \\
\text { (CO/AC) } \\
\text { Exogone naidina } \\
(\mathrm{CO} / \mathrm{OC})\end{array}$ & $\begin{array}{l}\text { i Chaetozone setosa } \\
\text { (CO/PR) } \\
\text { Spiophanes bombyx } \\
\text { (CO/OC) } \\
\text { Prionospio fallax } \\
\text { (CO/OC) } \\
\text { Streptosyllis } \\
\text { websteri (CO/OC) } \\
\text { Spio decoratus } \\
\text { (CO/OC) } \\
\text { Galathowenia } \\
\text { oculata (CO/PR) } \\
\text { Caulleriella alata } \\
\text { (CO/PR) } \\
\text { Exogones hebes } \\
\text { (CO/OC) } \\
\text { Paradoneis armata } \\
\text { (CO/AC) } \\
\text { Heteromastus } \\
\text { filiformis (CO/OC) } \\
\text { Euclymene oerstedi } \\
\text { (CO/AC) }\end{array}$ & $\begin{array}{l}\text { Spiophanes bombyx } \\
\text { (CO/OC) } \\
\text { Magelona filiformis } \\
\text { (CO/AC) } \\
\text { Capitella capitata } \\
\text { (CO/OC) } \\
\text { Paradoneis armata } \\
\text { (CO/OC) } \\
\text { Spio decoratus } \\
\text { (CO/OC) } \\
\text { Exogone hebes } \\
\text { (CM/OC) }\end{array}$ & $\begin{array}{l}\text { Exogone hebes } \\
\text { (CO/OC) } \\
\text { Spiophanes bom- } \\
\text { byx (VC/OC) } \\
\text { Capitella capitata } \\
\text { (CO/OC) } \\
\text { Prionospio fallax } \\
\text { (CO/OC) } \\
\text { Cossura pygodac- } \\
\text { tylata }(\mathrm{CO} / \mathrm{EX}) \\
\text { Pseudopolydora } \\
\text { sp. (CO/EX) } \\
\text { Prionospio } \\
\text { pulchra (VC/PR) } \\
\text { Spio decoratus } \\
\text { (CO/OC) }\end{array}$ \\
\hline $\mathrm{S}$ & $28.58 \pm 16.97$ & $34.47 \pm 2.12$ & $27.89 \pm 3.00$ & $42.55 \pm 4.79$ & $19.21 \pm 4.59$ & $24.33 \pm 8.54$ \\
\hline $\mathrm{N}$ & $608.57 \pm 364.87$ & $1903.10 \pm 120.21$ & $854.72 \pm 222.17$ & $729.46 \pm 367.60$ & $227.71 \pm 236.69$ & $524.37 \pm 607.32$ \\
\hline$H^{\prime}$ & $3.28 \pm 1.29$ & $3.58 \pm 0.14$ & $2.48 \pm 0.70$ & $4.11 \pm 0.12$ & $3.00 \pm 0.26$ & $2.95 \pm 1.12$ \\
\hline $\mathrm{J}$ & $0.69 \pm 0.15$ & $0.70 \pm 0.02$ & $0.52 \pm 0.14$ & $0.76 \pm 0.03$ & $0.70 \pm 0.07$ & $0.65 \pm 0.18$ \\
\hline Depth (m) & $9.17 \pm 3.54$ & $10.4 \pm 2.10$ & $8.9 \pm 1.00$ & $7.50 \pm 0.60$ & $4.70 \pm 3.10$ & $2.91 \pm 0.82$ \\
\hline$\%$ TOM & 1.48 & $1.40 \pm 0.12$ & $2.18 \pm 0.11$ & $2.40 \pm 0.54$ & $2.15 \pm 0.30$ & $6.76 \pm 3.81$ \\
\hline$\% \mathrm{CO}_{3}=$ & 24.26 & $65.01 \pm 5.40$ & $80.89 \pm 1.57$ & $54.37 \pm 20.46$ & $39.30 \pm 7.16$ & $5.93 \pm 1.25$ \\
\hline $\mathrm{Q}_{50}(\mathrm{~mm})$ & 5.00 & $0.86 \pm 0.05$ & $0.33 \pm 0.02$ & $0.18 \pm 0.05$ & $0.11 \pm 0.03$ & $0.03 \pm 0.02$ \\
\hline$\%$ gravel & 94.23 & $9.28 \pm 1.43$ & $0.94 \pm 0.44$ & $5.27 \pm 3.29$ & $0.08 \pm 0.07$ & $0.73 \pm 1.20$ \\
\hline$\%$ sand & 5.74 & $90.05 \pm 2.34$ & $95.96 \pm 0.44$ & $89.29 \pm 4.07$ & $90.47 \pm 4.85$ & $26.27 \pm 4.81$ \\
\hline$\%$ silt/clay & 0.03 & $0.67 \pm 0.91$ & $3.10 \pm 0.05$ & $5.44 \pm 1.21$ & $9.45 \pm 4.81$ & $73.00 \pm 17.21$ \\
\hline Sorting & Moderate & Moderate & Moderate & Moderate & $\begin{array}{l}\text { Mod.-mod. well } \\
\text { sorted }\end{array}$ & Poor \\
\hline $\begin{array}{l}\text { Sedimentary } \\
\text { type }\end{array}$ & Gravel & Coarse sand & Medium sand & Fine to muddy sand & Fine to muddy sand & $\begin{array}{l}\text { Sandy mud to } \\
\text { mud }\end{array}$ \\
\hline
\end{tabular}

Licher and Mackie, 2000 (constant, elective) and Diopatra neapolitana delle Chiaje, 1841 (common, accessory) although these species appeared in low numbers.

Group C3 comprised four sites $(16,19,20,21)$ with muddy and poorly sorted sediments. Site 16 showed the highest organic matter and silt/clay content of the inlet, as well as the lowest species number and diversity. This assemblage was characterized by the presence of Cossura pygodactylata (constant, exclusive), Prionospio pulchra Imajima, 1990 (very common, preferential), Pseudopolydora sp. (constant, exclusive) and Melinna palmata Grube, 1870 (very common, elective), although Exogone hebes, Spiophanes bombyx and Capitella capitata exhibited a greater total dominance, mainly due to their abundance at site 21 .

\section{Species affinities}

Cluster and MDS analyses performed on the abundance data of the species considered as dominant in each site show the existence of five species groups at a 50\% similarity level (Fig. 2). Group 1 was comprised of species found in gravel bottoms (site group A). Group 2 included species only found in coarse and medium sands (site groups B1-B2). Group 3 consisted of species mostly found at sites located in the middle of the inlet, where coarse-medium sand bottoms change to medium-fine sand sediments, namely Streptosyllis websteri and Caulleriella bioculata (species common to both groups B2 and C1). Group 4 comprised the largest group of species, mainly found in groups $\mathrm{C} 1, \mathrm{C} 2$ and $\mathrm{C} 3$, including those present in fine sand sediments with a high content of 


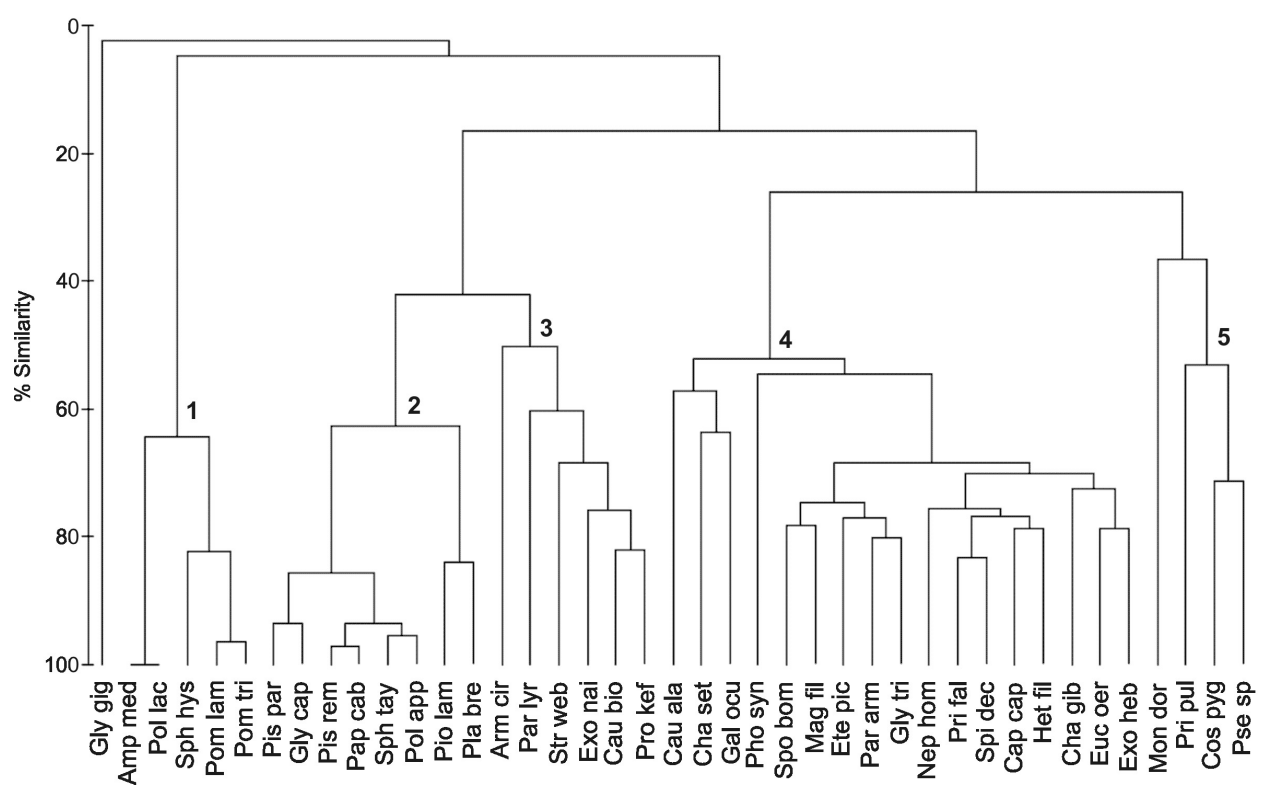

FIG. 2. - Dendrogram based on cluster analysis showing the classification of species with a numerical dominance $\geq 4 \%$ at any given site. Species codes: Amp med, Amphiglena mediterranea; Arm cir, Armandia cirrosa; Cap cap, Capitella capitata; Cau ala, Caulleriella alata; Cau bio, C. bioculata; Cha gib, Chaetozone gibber; Cha set, C. setosa; Cos pyg, Cossura pygodactylata; Ete pic, Eteone picta; Euc oer, Euclymene oerstedi; Exo heb, Exogone hebes; Exo nai, E. naidina; Gal ocu, Galathowenia oculata; Gly cap, Glycera capitata; Gly gig, G. gigantea; Gly tri, G. tridactyla; Het fil, Heteromastus filiformis; Mag fil, Magelona filiformis; Mon dor, Monticellina dorsobranchialis; Nep hom, Nephtys hombergi; Pap cab, Parapionosyllis cabezali; Par arm, Paradoneis armata; Par lyr, P. lyra; Pho syn, Pholoe synophthalmica; Pio lam, Pionosyllis lamelligera; Pis par, Pisione parapari; Pis rem, P. remota; Pla bre, Plakosyllis brevipes; Pol app, Polygordius appendiculatus; Pol lac, P. lacteus; Pom lam, Pomatoceros lamarcki; Pom tri, P.triqueter; Pri fal, Prionospio fallax; Pri pul, P. pulchra; Pro kef, Protodorvillea kefersteini; Pse sp, Pseudopolydora sp.; Sph hys, Sphaerosyllis hystrix; Sph tay, S. taylori; Spi dec, Spio decoratus; Spo bom, Spiophanes bombyx; Str web, Streptosyllis websteri.

medium sand (Galathowenia oculata, Chaetozone setosa, Caulleriella alata) and those with a greater abundance in muddier bottoms (Euclymene oerstedi (Claparède, 1863), Exogone hebes, Capitella capitata, Nephtys hombergi Savigny, 1818). Finally, Group 5 was composed of species found in muddy bottoms (site group C3), such as Pseudopolydora sp. and Cossura pygodactylata.

\section{Relationships between faunistic and abiotic data}

High correlations of selected environmental variables with faunistic data were obtained through the BIO-ENV procedure $\left(p_{\mathrm{w}}>0.70\right)$. The median grain size was the variable that showed the highest correlation through the Spearman rank correlation coefficient $\left(p_{\mathrm{w}}: 0.75\right)$, followed by very fine sand $\left(p_{\mathrm{w}}\right.$ : $0.67)$. The combinations of median grain size, coarse sand, medium sand, fine sand and very fine sand showed the highest correlation values $\left(p_{\mathrm{w}}\right.$ $>0.78$ ). As in the case of BIO-ENV global results, the forward selection of CCA selected median grain size, gravel, medium sand and very fine sand as the variables which significantly accounted for $(\mathrm{p}<0.01)$ most of the variance in species data (1.55 of a total variance of 2.21).
The graphic representation of CCA showed an ordination of sites similar to that obtained through MDS (Fig. 3). Axes I and II were the most impor-

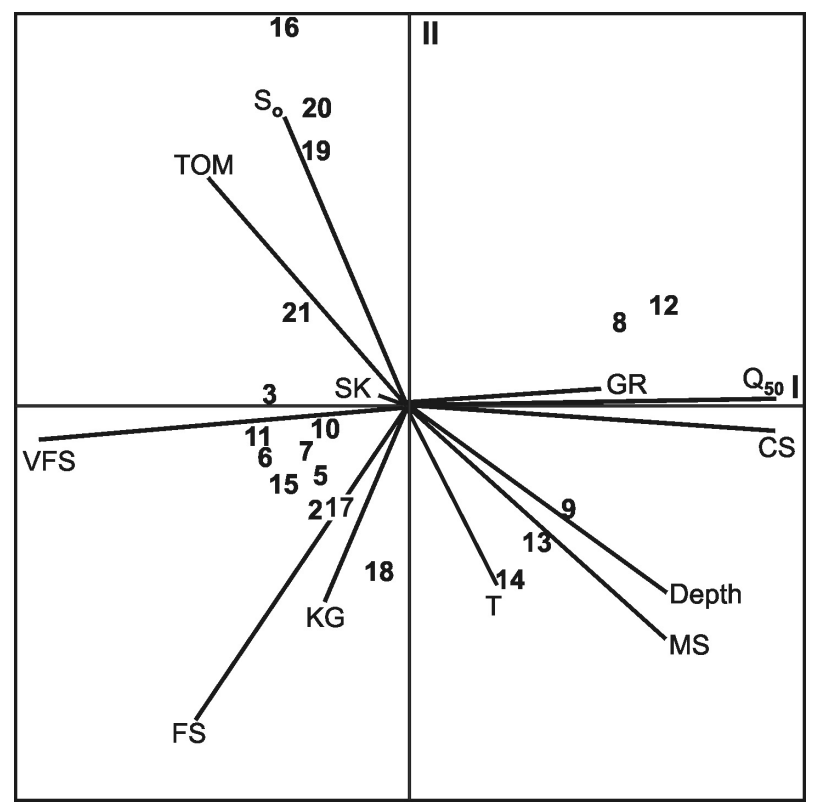

FIG. 3. - Canonical correspondence analysis (CCA) ordination of sites and environmental variables relative to axes I and II. GR, gravel; CS, coarse sand; MS, medium sand; FS, fine sand; VFS, very fine sand; $\mathrm{Q}_{50}$, median grain size; $\mathrm{S}_{\mathrm{o}}$, sorting coefficient; $\mathrm{KG}$, kurtosis; SK, skewness; TOM, total organic matter; T, temperature. 
tant, showing the highest eigenvalues $(0.814$ and 0.338 , respectively) and accumulated $43.4 \%$ of the species variance and $52.0 \%$ of species-environment variance. Median grain size, coarse sand and very fine sand showed the highest correlations with axis I. Cluster site groups dominated by coarser granulometric fractions appear distributed to the right of the graph, while fine sand-muddy assemblages appear to the left, following a gradient defined by a decrease in median grain size (Fig. 3).

\section{DISCUSSION}

In spite of its small extent, the study area showed a diverse polychaete fauna. The total number of species was remarkably higher than that found in other Galician rías, which usually have muddier sediments and higher organic matter content (LópezJamar, 1978, 1981). Anoxic conditions and organic enrichment related to human activities are frequently reported from those bottoms, which show a reduction in number of species and a numerical dominance by species such as Capitella capitata, Malacoceros fuliginosus (Claparède, 1870) and Spiochaetopterus costarum (Claparède, 1870) (López-Jamar, 1978; Rodríguez-Castelo and Mora, 1984).

Distribution of soft-bottom polychaetes and infauna in general have been related to sediment characteristics, depth, temperature and salinity (Gray, 1974; Hutchings, 1998). Simboura et al. (2000) found that type of substratum and hydrodynamism are the major factors controlling polychaete spatial distribution in shallow waters. Our results suggest that sedimentary composition plays a major role in determining polychaete distribution in the area studied. On the contrary, depth was not noted as an important factor, perhaps due to the shallow nature of the area. Fitzhugh (1984) pointed out that continental shelf polychaete assemblages respond to changes in depth-related parameters such as bottom-water variability, sedimentary stability and food availability rather than to depth itself. Likewise, these parameters are mostly controlled by wave action and tidal current speed over shallow bottoms (Cabioch, 1968). In the Ensenada de Baiona, sediment distribution is clearly related to wave propagation patterns in the area (Alejo et al., 1999), because a gradient in grain size exists from the ocean-exposed outer to the sheltered inner areas. In our case, distribution of polychaete assemblages follows this sedimentary gradient.
Aside from the two gravel sites, two major faunistic assemblages were determined through multivariate analyses. Coarser sandy sediments (group B) had a polychaete fauna mostly dominated by syllids and pisionids, with the former also having a large number of species. Clean coarse sandy sediments are known to provide a wide range of interstitial spaces which constitute a suitable habitat for these families (Dauvin, 1988; Westheide, 1990). Finer sandy sediments (groups C1, C2) showed a greater numerical dominance by deposit-feeders such as spionids, cirratulids and capitellids. Even though dominance by spionids has been considered by some as an indication of polluted environments, their abundance in these sediments should not be taken as a symptom of anthropogenic perturbation. In fact, their reproductive and feeding plasticity makes them good colonizers of different kinds of environments (Herrando-Pérez et al., 2000), taking advantage of the natural supply of organic matter to sediments under non-polluting conditions (RodríguezVilanueva et al., 2003).

The greatest number of species and diversity values were recorded in the fine sands belonging to group $\mathrm{C} 1$. These sites showed a greater sedimentary structural complexity due to a mixing of the fine and medium sand fractions with the presence of a small but significant percentage of gravels (mostly broken shells). On the other hand, group C2 had sediments dominated by finer sandy fractions as well as a lower number of species and abundance. Simboura et al. (2000) also found high polychaete diversity values and maximal number of species in mixed sandy bottoms with biogenic fragments. In fact, greater faunal diversity is mostly associated with greater grain size heterogeneity, in contrast to more homogenous, coarse or fine sediments (Gray, 1974; Nicolaidou and Papadopolou, 1989), which have a smaller diversity of microhabitats.

The lowest number of species and diversity in the inlet was detected at site 16, a muddy bottom in the harbour area with the highest organic content, which could be considered as an impoverished facies of the mud assemblage (group C3). The deposit-feeder Cossura pygodactylata was the dominant species on this site ( $>70 \%$ of the total polychaete abundance). Bachelet and Laubier (1994) recorded high densities of this species in organically enriched shallow softbottoms and Glémarec and Grall (2000) regarded it as an opportunistic species whose presence indicates hypoxic-anoxic conditions. Thus, it can be suspect- 
ed that the harbour environment is experiencing the effects of organic enrichment and a consequent reduction in faunal diversity. This situation is not surprising, because the construction of the harbour jetty has already altered current dynamics into the inlet and the natural patterns of sedimentary deposition (Alejo and Vilas, 1987). López-Jamar and Mejuto (1985) reported a similar process in the sandy Ría da Coruña after the construction of harbour facilities. These changes, in addition to other human activities such as disposal of organic sewage, result in a progressive increase of finer granulometric fractions and organic matter around these areas, which would imply a substitution of the original fauna by opportunistic species with a greater preference for muddier sediments.

\section{ACKNOWLEDGEMENTS}

The authors wish to thank F.J. Cristobo, C. Olabarría and P. Reboreda for their invaluable help with sample collection, A. Jackson for correcting the English and J.C. Dauvin and two anonymous referees for their constructive criticism on the early draft of the manuscript.

\section{REFERENCES}

Abella, F.E., J.M. Parada and J. Mora. - 1996. Relationship between the macrobenthic community structure and the presence of mussel rafts culture in the Ria de Vigo (NW Iberian Peninsula). Crangon, 1: 111-118.

Alejo, I., W.E.N. Austin, G. Francés and F. Vilas. - 1999. Preliminary investigations of the recent Foraminifera of Baiona Bay, N.W. Spain. J. Coast. Res., 15: 413-427.

Alejo, I. and F. Vilas. - 1987. Dinámica litoral y evolución histórica de la Ensenada de Bayona (Pontevedra). Thalassas, 5: 21-32.

Bachelet, G. and L. Laubier. - 1994. Morphology, ecology and juvenile development of Cossura pygodactylata Jones (Polychaeta, Cossuridae) in Arcachon Bay, SW France, with a reassessment of the geographical distribution of C. pygodactylata and C. soyeri Laubier. Mém. Mus. Natn. Hist. Nat., 162: 355-369.

Bellan, G. - 1967. Pollution et peuplements benthiques sur substrats meubles dans la région de Marseille. Première partie. Le secteur de Cortiou. Rev. Intern. Océanogr. méd., 6(7): 53-87.

Cabioch, L. - 1968. Contribution a la connaissance des peuplements benthiques de la Manche occidentale. Cah. Biol. Mar., 9 (Suppl.): 493-720.

Clarke, K.R. and R.M. Warwick. - 1994. Changes in Marine Communities: an approach to statistical analyses and interpretation. Natural Environment Research Council, U.K.

Dauvin, J.-C. - 1988. Structure et organisation trophique du peuplement des sables grossiers à Amphioxus lanceolatus-Venus fasciata de la baie de Morlaix (Manche occidentale). Cah. Biol. Mar., 29: 163-185.

Field, J.G., K.R. Clarke and R.M. Warwick. - 1982. A practical strategy for analysing multispecies distribution patterns. Mar. Ecol. Prog. Ser., 8: 7-52.

Fitzhugh, K. - 1984. Temporal and spatial patterns of the poly- chaete fauna on the central Northern Gulf of Mexico continental shelf. In: P.A. Hutchings (ed.), Proceedings of the First International Polychaete Conference, Sydney, pp. 211-225. The Linnean Society of New Wales.

Glémarec, M. and J. Grall. - 2000. Les groupes écologiques et zoologiques d'invertébrés marins face aux dégradations de l'environnement côtier. Bull. Soc. Zool. Fr., 125: 37-48.

Gray, J.S. - 1974. Animal-sediment relationships. Oceanogr. Mar. Biol. Ann. Rev., 12: 223-261.

Gray, J.S. and F.B. Mirza. - 1979. A possible method for detecting pollution induced disturbance on marine benthic communities. Mar. Pollut. Bull., 10: 142-146.

Herrando-Pérez, S., G. San Martín and J. Núñez. - 2000. Polychaete patterns from an oceanic island in the eastern Central Atlantic: La Gomera (Canary Archipelago). Cah. Biol. Mar., 42: 275-287.

Hutchings, P. - 1998. Biodiversity and functioning of polychaetes in benthic sediments. Biodiv. Conserv., 7: 1133-1345.

Junoy, J. and J.M. Viéitez. - 1989. Cartografía de los sedimentos superficiales de la Ría de Foz (Lugo). Thalassas, 7: 9-19.

López-Jamar, E. - 1978. Macrobentos infaunal de la Ría de Pontevedra. Bol. Inst. Esp. Oceanogr., 4(264): 111-130.

López-Jamar, E. - 1981. Spatial distribution of the infaunal benthic communities of the Ría de Muros, North-West Spain. Mar. Biol., 63: 29-37.

López-Jamar, E. and R.M. Cal. - 1990. El sistema bentónico de la zona submareal de la ría de Vigo. Macroinfauna y microbiología del sedimento. Bol. Inst. Esp. Oceanogr., 6: 49-60.

López-Jamar, E., J. Iglesias and J.J. Otero. - 1984. Contribution of infauna and mussel-raft epifauna to demersal fish diets. Mar. Ecol.Prog. Ser., 15: 13-18.

López-Jamar, E. and J. Mejuto. - 1985. Bentos infaunal en la zona submareal de la ría de La Coruña. I. Estructura y distribución espacial de las comunidades. Bol. Inst. Esp. Oceanogr., 2: 99-109.

Moreira, J., J. Parapar and J.S. Troncoso. - 2000a. On the presence of Prionospio pulchra (Polycheta: Spionidae) in the Atlantic Ocean. Cah. Biol. Mar., 41: 233-239.

Moreira, J., P. Quintas and J.S. Troncoso. - 2000b. Pisione parapari n. sp., a new pisionid from the North-East Atlantic. Ophelia, 52: 177-182.

Nicolaidou, A. and K.N. Papadopoulou. - 1989. Factors affecting the distribution and diversity of polychaetes in Amvrakikos Bay, Greece. P.S.Z.N.I.: Mar. Ecol., 10: 193-204.

Parapar, J., G. San Martín and J. Moreira. - 2000. Parapionosyllis cabezali, a new species of Exogoninae (Polychaeta: Syllidae) from Spain. Proc. Biol. Soc. Wash., 113: 526-531.

Planas, M. - 1986. Poliquetos de los fondos rocosos de las Islas Cíes (NW España). Trab. Comp. Biol., 13: 71-88.

Rodríguez-Castelo, E. and J. Mora. - 1984. Introducción al estudio de una dinámica de poblaciones bentónicas en fangos infralitorales orgánicamente enriquecidos (Ría de Pontevedra. NW de España). Cuadernos da Área de Ciencias Mariñas, Seminario de Estudos Galegos, 1: 291-302.

Rodríguez-Villanueva, V., R. Martínez-Lara and V.M. Zamora. 2003. Polychaete community structure of the northwestern coast of Mexico: patterns of abundance and distribution. Hydrobiologia, 496: 385-399.

Simboura, N., A. Nicolaidou and M. Thessalou-Legaki. 2000. Polychaete communities of Greece: An ecological overview. P.S.Z.N.I.: Mar. Ecol., 21: 129-144.

ter Braak, C.J.F. - 1988. Canoco - a Fortran program for canonical community ordination by partial, detrended, canonical correspondence analysis, principal component analysis and redundancy analysis. Agricultural Mathematics Group, Ministry of Agriculture and Fisheries, Ithaca, N.Y.

Trask, P.D. - 1932. Origin and environment of source sediments of petroleum. Houston Gulf Publications Co., Houston.

Viéitez, J.M. - 1976. Ecología de Poliquetos y Moluscos de la playa de Meira (Ría de Vigo). I. Estudio de las comunidades. Inv. Pesq., 40: 223-248.

Westheide, W. - 1990. Polychaetes: interstitial families. Synopses of the British Fauna (New series), 44. Linnean Society of London and Estuarine and Coastal Sciences Association, Universal Book Services/ Dr. W. Backhuys, The Netherlands.

Received July 9, 2004. Accepted May 11, 2005. 